\title{
Systematics of the fusion cross-section in heavy ion reactions
}

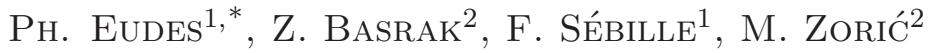 \\ and V. DE LA MotA ${ }^{1}$
}
${ }^{1}$ SUBATECH, EMN-IN2P3/CNRS-Université de Nantes, P.O.Box 20722, F-44307 Nantes cedex 3, France
${ }^{2}$ Ruder Bošković Institute, P.O.Box 118, HR-10 002 Zagreb, Croatia

\begin{abstract}
We study the energy dependence of the fusion cross-section with the goal of understanding the evolution of the underlying reaction mechanism as a function of input channel parameters and the causes of its (non-)disappearance. The heart of this work is a systematic and as wide as possible overview of measured cross-sections. Normalized by the reaction cross-section and plotted as a function of the available energy per nucleon corrected for the Coulomb barrier, fusion crosssection show a rather universal behavior leading to a disappearance of the fusion around $10 \mathrm{~A} \mathrm{MeV}$, except in the case of very asymmetric systems for which incomplete fusion persists and tends towards a constant value that can be explained in the frame of a simple geometrical approach. These trends are confirmed by the semiclassical Landau-Vlasov transport calculations.
\end{abstract}

In heavy ion collisions, one observes complete fusion $(\mathrm{CF})$ and/or incomplete fusion (IF) mechanisms of the two partners in the most central reactions over an impact parameter range whose width depends strongly on the incident energy [1]. From the Coulomb barrier to $6-8 A \mathrm{MeV}$, only $\mathrm{CF}$ occurs and it accounts for the largest part of the reaction cross section.

*E-mail: eudes@subatech.in2p3.fr

This is an Open Access article distributed under the terms of the Creative Commons Attribution License 2.0, which permits unrestricted use, distribution, and reproduction in any medium, provided the original work is properly cited. 


\section{EPJ Web of Conferences}

Between 6-8 and 20-25A MeV, both CF and IF mechanisms coexist. IF increases while $\mathrm{CF}$ fades gradually and disappear around $20 A \mathrm{MeV}$. Between 20-25 and 40A MeV, IF still exists but represents only a smaller and smaller portion of the reaction cross section. Beyond the Fermi energy, the vanishing of fusion is reported for light systems ( [2] and references therein) while it seems to persist for strongly asymmetric systems up to $155 \mathrm{~A} \mathrm{MeV} \mathrm{[3].}$

To study the evolution of fusion processes and quantify them by different entrance channel parameters (incident energy, total system mass and mass asymmetry) up to very recently a number of dedicated experiments has been carried out to extract the CF and/or IF cross sections [4]. Most of them are inclusive and fusion is then evidenced by the detection of an evaporation residue (ER) and/or fission fragments with the expected kinematic properties. Indeed, $\mathrm{CF}$ and $\mathrm{IF}$ are characterized by the formation of a compound or quasi-compound nucleus (that means a single nuclear source) which equilibrates all its degrees of freedom and which de-excites standardly by evaporation process or if allowed, by equilibrated fission. Other de-excitation mechanisms could coexist and explain the disappearance of fusion above the Fermi energy, like multi-fragment emission from a single equilibrated source. Measurements taking into account both single and multiple-fragment exit channels are rather scarce [2] and fusion crosssection shown in this paper corresponds almost exclusively to the sum of fusion-fission and fusion-evaporation cross sections.

The goal of this work is to revisit the fusion excitation function by providing as complete as possible systematics on measured fusion cross-sections (FCS) including summed up CF and IF cross-sections. Such a work was initiated by the INDRA Collaboration who published a fusion excitation function for 7 light nearly symmetric systems [2]. In the present study, the systematics has been considerably enlarged and 168 cross-section data points (corresponding to 57 different systems) have been collected from measurements undertaken over the last forty years. This allows us to cover a span in total system mass $A_{\text {tot }}=A_{t}+A_{p}\left(A_{t}\right.$ and $A_{p}$ are target and projectile mass $)$ between 26 and 246, a system mass asymmetry $\mu=\left(A_{t}-A_{p}\right) /\left(A_{t}+A_{p}\right)$ in the range from 0 to $0.886, N / Z$ ratio in the range from 1 to 1.536 and a range of incident energy that lies between $4 A$ and $155 \mathrm{~A} \mathrm{MeV}$. (This work is not focusing on fusion aspects around the Coulomb barrier.) These raw FCS are displayed as a function of incident energy in the laboratory reference frame in figure 1.

The different systems are ordered by increasing mass $A_{\text {tot }}$. A color code (online) is used to differentiate between them: blue and green symbols for the lighter systems $\left(26<A_{\text {tot }}<116\right)$ and pink and red symbols for the heavier 


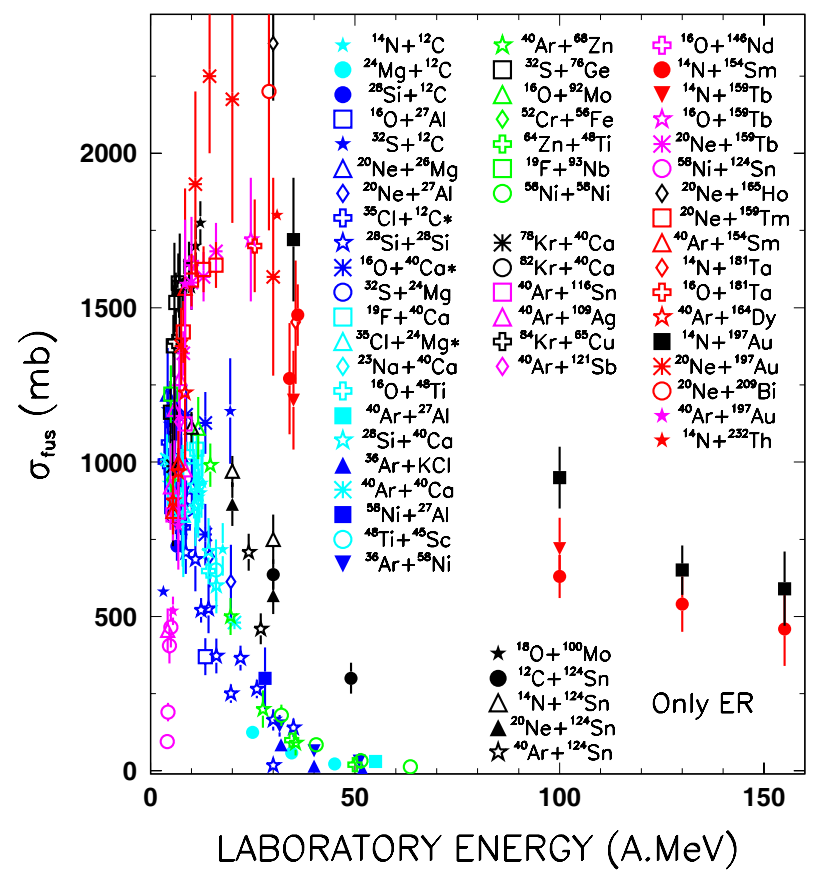

Figure 1: (Colour online.) Raw fusion cross sections plotted as a function of the laboratory energy per nucleon $E_{\mathrm{LAB}} /$ nucl.

ones $\left(146<A_{\text {tot }}<246\right)$. Black symbols are used to point out measurements for which in the original publication an overestimation of the fission component due to a mix between equilibrated fission and fast-fission is explicitly mentioned [3-6]. Black symbols are also used for the five systems (labeled by Only ER in figure) for which only evaporation cross-sections were measured whereas a more or less significant fission component was expected too $[7,8]$.

Available data can be clearly divided into the two sets corresponding exactly to the lighter systems and to the heavier ones. It is instructive to sort these data following both their mass asymmetry and their isospin. Looking carefully at the list of systems on the right part of figure 1, one finds easily that lighter systems are rather symmetric both in mass and isospin $(0<\mu<0.5$ and $1<N / Z<1.25)$ while, on the contrary, heavier systems are very asymmetric in mass $(\mu>0.75$ for most of them) and, as expected, more asymmetric in isospin $(N / Z>1.3)$. Thus, sorting FCS by total system mass, mass asymmetry or isospin is roughly equivalent taking into account available data. Therefore, a reliable conclusion on the role of the above entrance channel parameters cannot be drawn. 


\section{EPJ Web of Conferences}

From the first part of this work one can conclude that for the lighter symmetric systems FCS regularly diminishes with incident energy and disappears above 35-40 A MeV. Despite of the complete lack of the data between 30 and $100 A \mathrm{MeV}$ one may conclude that for the heavier asymmetric systems FCS increases up to $20 \mathrm{~A} \mathrm{MeV}$, then decreases and persists up to incident energies as high as $155 \mathrm{~A} \mathrm{MeV}$. Let us note that there is only one experiment reported above $100 A \mathrm{MeV}$ [3]. Another lack of data concerns medium mass asymmetry systems for which very few data exist and new ones would be welcome.

The next step in this analysis is to normalize the fusion cross-section by the reaction cross section and to express them relative to the available energy corrected for the Coulomb barrier $\left(E_{\text {available }}-B_{C}\right) / n u c l$ to ease comparison of various systems. To calculate reaction cross-section, there exist several parameterizations (see e.g. Geant4 reference manual) all based on the strong absorption model. We used both the Kox and Tripathi formulae $[9,10]$, Kox formula being modified in order to extend its applicability down to a few $A \mathrm{MeV}$. Both formulae give very similar results except below $\left(E_{\text {available }}-B_{\mathrm{C}}\right) /$ nucl $<1 A \mathrm{MeV}$ where obvious discrepancies are evidenced. In figures 2 and 3 are displayed normalized FCS using Tripathi prescription to the lighter (nearly) symmetric systems and to the heavier (asymmetric ones), respectively. All data are kept, but a grey (yellow online) zone shows uncertain data below the $1 A \mathrm{MeV}$ threshold.

These figures call for several comments. Instead of getting a single general behavior, plotted FCS strongly suggest the existence of two branches: a lighter symmetric one and a heavier asymmetric one. When only the lighter symmetric systems are considered (Fig. 2), apart from a few points corresponding to $\mathrm{N}+\mathrm{C}$ and $\mathrm{S}+\mathrm{C}$ systems, normalized FCS follow a universal law which can be fitted by an exponential function. Fusion then disappears at a value very close to $10 \mathrm{~A} \mathrm{MeV}$, expressed in the centre-of-mass energy corrected for the Coulomb barrier. To probe the disappearance of fusion, central ${ }^{36} \mathrm{Ar}+{ }^{36} \mathrm{Ar}$ collision calculations have been performed with the Landau-Vlasov model $[11,12]$ using the Gogny force and the free nucleonnucleon cross section $\sigma_{\mathrm{NN}}^{\mathrm{m}}$ with its empirical energy and isospin dependence for incident energies ranging from $25 \mathrm{~A} \mathrm{MeV}$ up to $40 \mathrm{AMeV}$. As the incident energy increases, one observes the formation of a more and more elongated composite system. Below around $30 \mathrm{~A} \mathrm{MeV}$, one obtains an incomplete fusion nucleus after a quite long time. Above, the calculations are in agreement with the experimental vanishing of fusion and provide an explanation: the elongated composite system finally gives rise to two nuclei having kept a 


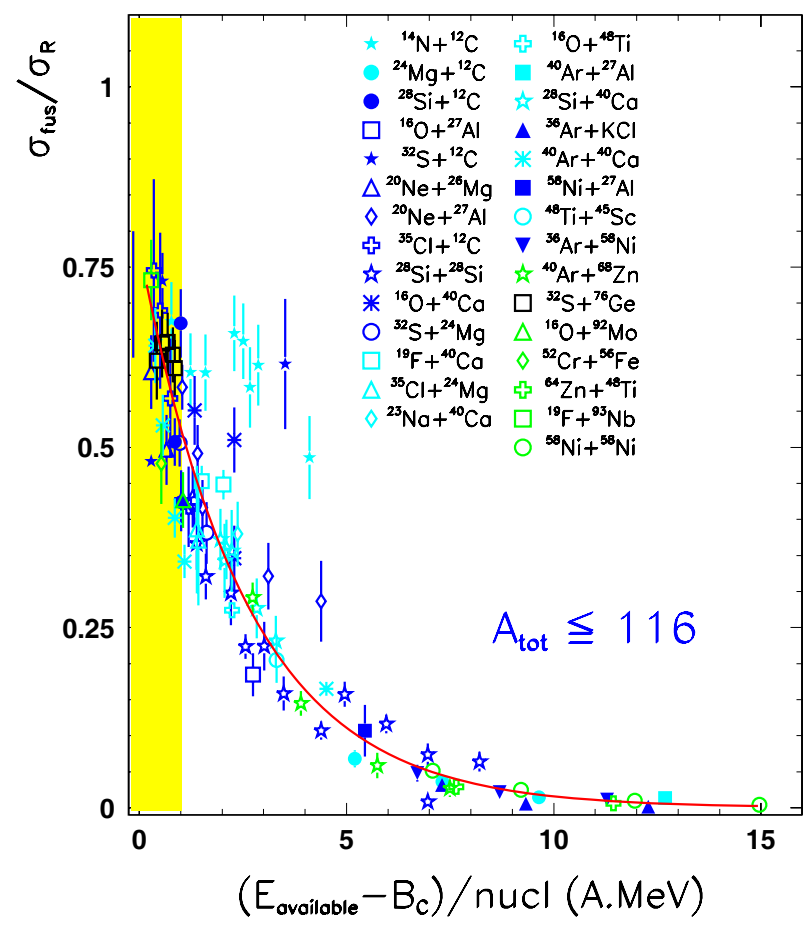

Figure 2: (Colour online.) Normalized fusion cross sections for lighter and rather symmetric systems plotted as a function of the available energy corrected for the Coulomb barrier.

strong memory of the entrance channel and a transparency phenomenon occurs. At this incident energy, all the reaction cross section is then of binary nature. We have already shown many years ago that the energy at which binary process is observed even in the most central collisions strongly depends on the nucleon-nucleon cross section used in the model [13].

Regarding the heavier asymmetric systems (Fig. 3), FCS also decreases but, as discussed above, by a first less convincing branch of systems labeled by Only ER and those following a second branch leading to a fairly constant value at the highest energies. In order to understand the persistence of fusion in this case, we performed simulations for the ${ }^{14} \mathrm{~N}+{ }^{154} \mathrm{Sm}$ system at $150 \mathrm{~A} \mathrm{MeV}$ over a large impact parameter range. In peripheral reactions, one observes a pre-equilibrium emission coming from the overlapping zone and two nuclei are formed in the exit channel. For impact parameters smaller than $b_{\max }$, i.e. those leading to a complete overlap of the projectile by the target, one obtains the formation of a massive ER accompanied by an abundant pre-equilibrium emission in the forward direction. This picture 


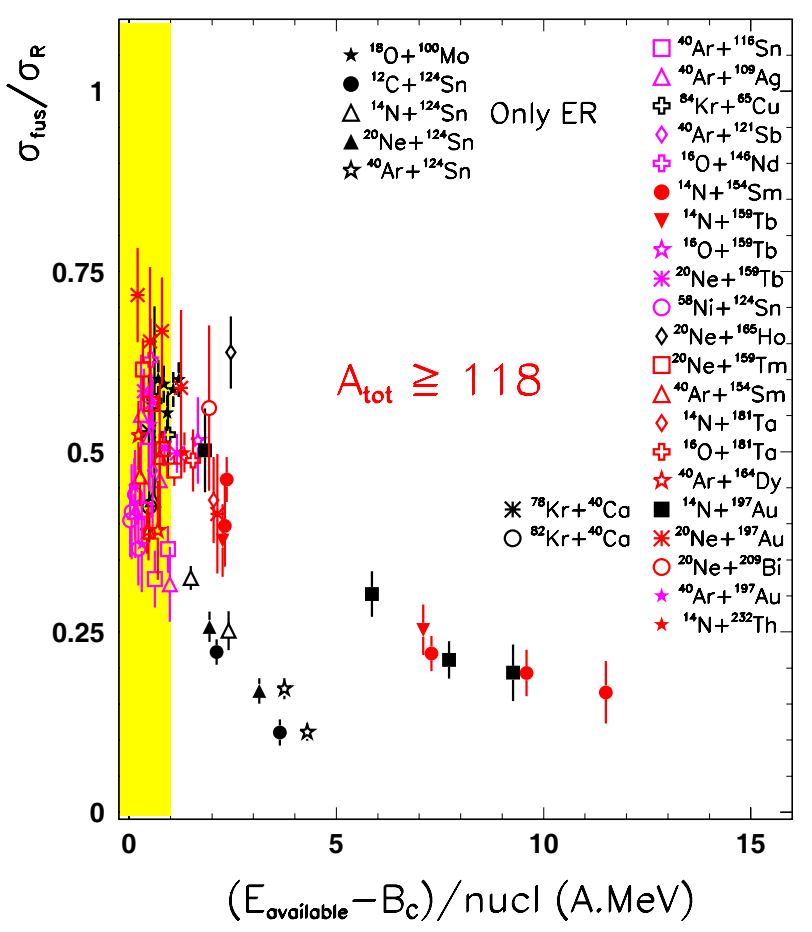

Figure 3: (Colour online.) Same as figure 2 for heavier asymmetric systems.

reminds the geometrical massive transfer model in which the overlapping part of the projectile fuses with the target while the rest is emitted either as pre-equilibrium or quasi-projectile. In this picture, fusion cross-section has to be comparable to the cross-section corresponding to a complete overlap. In a very simple picture, it can be parameterized as:

$$
\sigma_{\text {fus }}=\pi b_{\max }^{2}=\pi\left(R_{t}-R_{p}\right)^{2}=\pi r_{0}^{2}\left(A_{t}^{1 / 3}-A_{p}^{1 / 3}\right),
$$

where $R_{p}\left(R_{t}\right)$ is projectile (target) radius. Assuming a simple formula for the reaction cross section

$$
\sigma_{\text {reac }}=\pi r_{0}^{2}\left(A_{t}^{1 / 3}+A_{p}^{1 / 3}\right)
$$

one gets

$$
\frac{\sigma_{\text {fus }}}{\sigma_{\text {reac }}}=\left[1-\frac{2}{1+\left(\frac{A_{t}}{A_{p}}\right)^{1 / 3}}\right]^{2}=\left[1-\frac{2}{1+\left(\frac{1+\mu}{1-\mu}\right)^{1 / 3}}\right]^{2}
$$


Eq. (3) indicates that at high energies, normalized fusion cross-section tends effectively towards a constant value only depending on the mass asymmetry $\mu$. Numerical applications give $14.4 \%$ and $17 \%$ respectively for ${ }^{14} \mathrm{~N}+{ }^{154} \mathrm{Sm}$ and ${ }^{14} \mathrm{~N}+{ }^{197} \mathrm{Au}$ systems, that are in good agreement with experimental data.

Available experimental data allowed building the fusion excitation function. One may draw the following main conclusions:

1 - For lighter symmetric systems, normalized FCS decreases exponentially and disappears around $10 \mathrm{~A} \mathrm{MeV}$.

2 - For heavier asymmetric systems, it seems to decrease following a second branch leading to a constant value depending on the system mass asymmetry.

3 - Additional experimental data would be required especially for systems of medium and high mass asymmetries in order to corroborate the point 2 .

4 - Describing all the observed trends of the fusion excitation function could be a real challenge for dynamical models.

\section{References}

[1] R. Planeta , Int. J. Mod. Phys. E 15, 973 (2006).

[2] P. Lautesse et al. , Eur. Phys. J. A 27, 349 (2006).

[3] A.A. Songzoni et al., Phys. Rev. C 53, 243 (1996).

[4] G. Ademardet al. , Phys. Rev. C 83, 054619 (2011).

[5] G. Guillaume et al. , Phys. Rev. C 26, 2458 (1982).

[6] H.C. Britt et al. , Phys. Rev. C 13, 1483 (1976).

[7] H. Nifenecker et al. , Nucl. Phys. A 447, 533c (1985).

[8] M.P. Kelly et al. , Phys. Rev. C 56, 3201 (1997).

[9] S. Kox et al. , Phys. Rev. C 35, 1678 (1987).

[10] A. Tripathi et al. , NASA Technical Paper, 3621 (1997).

[11] C. Grégoire et al. , Nucl. Phys. A465, 317 (1987).

[12] F. Sébille et al. , Nucl. Phys. A 501, 137 (1989).

[13] Z. Basrak and Ph. Eudes, Acta. Phys. Polonica B 31, 361 (2000). 